\title{
Assessment of Systemic Immune Inflammation Index to Predict SARS-CoV-2 Infection
}

\author{
Dea Noviana Pramantik ${ }^{1}$, Dwi Aryani ${ }^{2}$ \\ ${ }^{1}$ Grhasia Mental Hospital, Yogyakarta, Indonesia. E-mail: novianadea@gmail.com \\ ${ }^{2}$ Sleman General Hospital, Yogyakarta, Indonesia
}

\begin{abstract}
Coronavirus Disease 2019 (COVID-19) is an infectious disease caused by Severe Acute Respiratory Syndrome Coronavirus 2 (SARS-CoV-2) and has become a major health problem worldwide. Inflammation plays a vital role in the pathophysiology of COVID-19. Systemic Immune Inflammation Index (SII) is an index obtained from calculating the platelets counts, neutrophils, and lymphocytes, which can indicate the inflammation status and immunity. This study aimed to determine the potential of SII as a predictor of SARS-CoV-2 infection in suspected COVID-19 subjects. A retrospective study was carried out by obtaining medical record data in June 2020 at Sleman General Hospital. An unpaired T-test or the Mann-Whitney test was used to determine the statistical difference. A Receiver Operating Characteristic (ROC) curve was generated and used to get the cut-off values. Bivariate analysis was performed using Chi-Square. There were 84 subjects consisting of $46(54.8 \%)$ males and $38(45.2 \%)$ females with a mean age of $42.4 \pm 16.356$ years. There was a significant difference in the neutrophils count $(p=0.045)$, monocytes $(p=0.001)$, and eosinophils $(p=0.037)$ between subjects with positive and negative SARS-CoV-2 PCR. The median SII in the positive and negative SARS-CoV-2 PCR group was 780.12 (301.21-2178.90)x103/ $\mu \mathrm{L}$ and 584.14 (117.79-1933.87) $\times 103 / \mu \mathrm{L}(\mathrm{p}=0.045)$, respectively. Bivariate analysis showed significant results at SII $>705 \times 103 / \mu \mathrm{L}$ in suspected COVID-19 patients to obtain a positive SARS-CoV-2 PCR result with Odds Ratio (OR) of 4.00 (95\% CI 1.580-10.127), $p=0.003$. Patients with suspected SARS-CoV-2 infection with high SII levels had a greater risk of a positive SARS-CoV-2 in PCR test.
\end{abstract}

Keywords: COVID-19, SARS-CoV-2, systemic immune inflammation index

\section{INTRODUCTION}

Coronavirus Disease 2019 (COVID-19) is an infectious disease caused by Severe Acute Respiratory Syndrome Coronavirus 2 (SARS-CoV-2). This disease has become a significant health problem worldwide since its first discovery in Wuhan, China, in December 2019. The World Health Organization (WHO) has designated COVID-19 as a pandemic in March 2020. A WHO report on June 28, 2020, had found 10 million confirmed cases with 500.000 death. The COVID-19 case in Indonesia continues to increase; therefore, comprehensive handling is needed in case management. ${ }^{1,2}$

The clinical presentation of patients with COVID-19 varies widely. Some infected people don't show any symptoms, although others can have clinical features like fever, fatigue, and respiratory symptoms such as cough, sore throat, runny nose, and anosmia. In addition, multi-organ dysfunction, sepsis, acute respiratory failure, and death-causing shock are found in severe cases. The use of efficient and straightforward laboratory tests in the early stages of handling of suspected COVID-19 patients is needed to reduce morbidity and disease mortality. ${ }^{13,4}$

The gold standard for diagnosing COVID-19 is the Polymerase Chain Reaction (PCR) to detect SARS-CoV-2. Routine laboratory tests to detect infection or inflammation, such as $\mathrm{C}$-reactive protein, procalcitonin, or complete blood counts, including leukocyte differential counts, can be considered in patients with suspected SARS-CoV-2 disease. Research showed that hematological parameters play an essential role in the triage and management of COVID-19. COVID-19 patients show abnormal results in hematological tests such as lymphopenia, neutrophilia, and thrombocytopenia. Evaluation of laboratory tests that are widely available, easy, and inexpensive will help the management of suspected COVID-19 patients. ${ }^{3-6}$

Inflammation plays a vital role in the pathophysiology of COVID-19. Therefore, the component of complete blood counts results can be used as markers of systemic inflammation.

The Systemic Immune Inflammation Index (SII) was introduced in 2014 by Hu et al. to predict the 
prognosis in hepatocellular carcinoma patients after surgery. Increased SII was significantly associated with vascular invasion, tumor size, and recurrence in hepatocellular carcinoma. Research shows that SII, calculated using data on the number of platelets, neutrophils, and lymphocytes, can indicate inflammation and immune status. The use of SII is relatively easy, widely available in many health care facilities, and inexpensive. The SII is calculated by multiplying platelet and absolute neutrophil count divided by absolute lymphocyte count. ${ }^{5,7-9}$

This study aimed to determine the profile and role of SII in predicting positive PCR results in subjects with suspected SARS-CoV-2 infection.

\section{METHODS}

A retrospective study was conducted at Sleman General Hospital, Yogyakarta, in June 2020. The data were obtained from the medical records of suspected COVID-19 patients who underwent PCR tests to identify SARS-CoV-2 infection. The epidemiological data, age, gender, and laboratory tests, such as complete blood counts, SII (platelets multiplied by NLR), and SARS-CoV-2 PCR results, were collected in this study. The blood sample was taken at the same time as the swab sampling. This study has received approval from the Health Research Ethics Committee of the Sleman General Hospital with number 180/2282.

The primary characteristics of research subjects were presented descriptively in terms of mean and standard deviation for data with normal distribution or median and the minimum-maximum value for data with the abnormal distribution. The normality test for continuous data was carried out by the Kolmogorov-Smirnov test, with a significance value of $p<0.05$. Categorical data were presented as proportions. An unpaired T-test and the Mann-Whitney test were used, based on the normality data distribution, to test the Bivariate mean analysis was performed using Chi-Square. A Receiver Operating Characteristic (ROC) curve was generated and used to get the cut-off values. The Area Under the Curve (AUC) was calculated. The 95\% of CI was then calculated, and $p<0.05$ was considered statistically significant. The data were processed using the SPSS software.

\section{RESULTS AND DISCUSSIONS}

In June 2020, 114 subjects who underwent SARS-CoV-2 PCR examination at Sleman General Hospital were found. A total of 30 subjects were excluded from analysis due to incomplete data in medical record files. There were 84 patients in this study, consisting of 46 (54.8\%) males and 38 (45.2\%) females with a mean age of $42.4 \pm 16.356$ years. The primary characteristics of research subjects are shown in Table 1.

Table 1. Characteristics of the research subject

\begin{tabular}{|c|c|}
\hline Variable & Value $n=84$ \\
\hline $\mathrm{Age}^{\mathrm{a}}$ & $42.4 \pm 16.356$ \\
\hline \multicolumn{2}{|l|}{ Gender, n (\%) } \\
\hline Male & $46(54.8 \%)$ \\
\hline Female & $38(45.2 \%)$ \\
\hline Hemoglobin, $(\mathrm{g} / \mathrm{dL})^{\mathrm{b}}$ & $13.90(10.2-17.3)$ \\
\hline Leucocyte $\left(\times 10^{3} / \mu \mathrm{L}\right)^{\mathrm{a}}$ & $8.25 \pm 2.55$ \\
\hline Neutrophil $\left(\times 10^{3} / \mu \mathrm{L}\right)^{a}$ & $5.08 \pm 1.90$ \\
\hline Lymphocyte $\left(x 10^{3} \mu \mathrm{L}\right)^{b}$ & $2.15(0.41-8.51)$ \\
\hline Monocyte $\left(x 10^{3} / \mu \mathrm{L}\right)^{\mathrm{b}}$ & $0.48(0.12-1.88)$ \\
\hline Eosinophil $\left(\times 10^{3} / \mu \mathrm{L}\right)^{b}$ & $0.09(0-1.1)$ \\
\hline Basophil $\left(\times 10^{3} / \mu \mathrm{L}\right)^{\mathrm{b}}$ & $0.03(0-0.11)$ \\
\hline Erythrocyte $\left(\times 10^{3} / \mu \mathrm{L}\right)^{\mathrm{a}}$ & $4.97 \pm 0.56$ \\
\hline Platelet $\left(\times 10^{3} / \mu \mathrm{L}\right)^{\mathrm{b}}$ & $295(77-690)$ \\
\hline SII $\left(\times 10^{3} / \mu \mathrm{L}\right)^{b}$ & $655.67(117.79-2178.90)$ \\
\hline
\end{tabular}

Table 2. Differences in hematology parameters based on SARS-CoV-2 PCR results

\begin{tabular}{|c|c|c|c|}
\hline Parameter & PCR Positive $n=33$ & PCR Negative $n=51$ & $\mathbf{p}$ \\
\hline Hemoglobin, $(g / d L)^{b}$ & $13.60(10.60-17.30)$ & $14.10(10.20-16.20)$ & 0.555 \\
\hline Leucocyte $\left(x 10^{3} / \mu \mathrm{L}\right)^{a}$ & $8.63 \pm 2.35$ & $8.01 \pm 2.66$ & 0.282 \\
\hline Neutrophil $\left(x 10^{3} / \mu \mathrm{L}\right)^{\mathrm{a}}$ & $5.59 \pm 1.76$ & $4.74 \pm 1.93$ & $0.045^{\star}$ \\
\hline Lymphocyte $\left(\times 10^{3} \mu \mathrm{L}\right)^{\mathrm{b}}$ & $1.83(1.20-6.15)$ & $2.18(0.41-8.51)$ & 0.108 \\
\hline Monocyte $\left(\times 10^{3} / \mu \mathrm{L}\right)^{\mathrm{b}}$ & $0.57(0.33-1.88)$ & $0.44(0.12-1.67)$ & $0.001^{*}$ \\
\hline Eosinophil $\left(\times 10^{3} / \mu \mathrm{L}\right)^{b}$ & $0.06(0-1.10)$ & $0.11(0-0.98)$ & $0.037^{*}$ \\
\hline Basophil $\left(\times 10^{3} / \mu \mathrm{L}\right)^{\mathrm{b}}$ & $0.03(0.01-0.1)$ & $0.03(0-0.11)$ & 0.558 \\
\hline Erythrocyte $\left(\times 10^{3} / \mu \mathrm{L}\right)^{\mathrm{a}}$ & $4.99 \pm 0.63$ & $4.95 \pm 0.52$ & 0.765 \\
\hline Platelet $\left(\times 10^{3} / \mu \mathrm{L}\right)^{\mathrm{b}}$ & $296(129-676)$ & $294(77-690)$ & 0.819 \\
\hline SII $\left(x 10^{3} / \mu \mathrm{L}\right)^{\mathrm{b}}$ & $780.12(301.21-2178.90)$ & 584.14 (117.79-1933.87) & $0.045^{*}$ \\
\hline
\end{tabular}

andependent T-test; ${ }^{\mathrm{b}}$ Mann-Whitney test; $\mathrm{p}<0.05$ was significant 
The results of this study indicated that the median SII was $655.67(117.79-2178.9) \times 10^{3} / \mu \mathrm{L}$. Research conducted by Zhang et al. in Wuhan, China, on 82 cases of death caused by COVID-19 found that the median SII was 1966.1 (923.1-3206.5) $\times 10^{3} / \mu \mathrm{L}$ with $89.2 \%$ of them had SII $>500 \times 10^{3} / \mu \mathrm{L}$. In the 24 hours before death, lymphopenia (73.7\%), neutrophilia (100\%), and thrombocytopenia (63.2\%) were observed. These three parameters are components needed in calculating SII. ${ }^{10}$

In this study, an analysis of the differences between several hematological parameters was carried out based on the results of the SARS-CoV-2 $P C R$. There was no significant difference in leukocytes counts between subjects with positive and negative SARS-CoV-2 PCR with $p=0.282$. This result was different from several research results that have been published previously. Ferrari et al. found a significant difference in the number of leukocytes in the PCR positive group compared to the negative PCR group with $p<0.001 .{ }^{11}$ Another study by Cheng et al. also showed a significantly lower number of leukocytes in the PCR positive group. ${ }^{12}$

Table 2 shows a difference in the mean number of neutrophils counts between subjects with positive and negative SARS-CoV-2 PCR. The mean neutrophils count in subjects with positive SARS-CoV-2 PCR is higher than subjects with negative SARS-CoV-2 PCR results $(p=0.045)$. A significant difference was also found in the monocytes counts $(p=0.001)$ and eosinophils counts $(p=0.037)$. Ferrari et al. also found a significant decrease in the number of leukocyte differentials consisting of neutrophils, eosinophils, basophils, lymphocytes, and monocytes in subjects with positive COVID-19 compared to negative COVID-19. ${ }^{11}$

The lymphocyte count in this study was relatively lower in the PCR-positive group; however, this was not statistically significant. The mechanism of lymphopenia in COVID-19 patients is related to the ability of the virus to infect T-cells through the Angiotensin-Converting Enzyme 2 (ACE2) receptor and CD147 spike protein. This process will lead to an increase in regulatory $\mathrm{T}$-cells and a decrease in $\mathrm{CD}^{+}$, $\mathrm{CD} 4^{+}, \mathrm{CD} 8^{+} \mathrm{T}$ lymphocytes, which in turn will be a risk factor for cytokine storm, which will cause multi-organ failure.

There was no significant difference in the number of platelets in the positive PCR group compared to the negative PCR with $p=0.819$. The interaction between activated platelets and vascular endothelial cells will cause inflammation and microcirculation disorders. Decreased platelets are associated with thrombosis and inflammation. ${ }^{13}$
Research on indicators of infection and inflammation using complete blood count test results continues to grow. Several parameters have been studied for the diagnosis and prognosis of various cases of infection and inflammation or malignancies, including SII. The systemic immune inflammation index reflects three hematologic markers that play a role in the inflammatory process. High platelet count and neutrophils indicate inflammation, while low lymphocytes indicate uncontrolled inflammatory processes. The SII test is widely used to predict prognosis in various cases of malignancy. In addition, there was also an association between malignancy, inflammation, sepsis, and clinical outcomes with SII. ${ }^{8,913-15}$

This study found a significant difference in SII between the positive and negative SARS-CoV-2 $P C R$ groups with $p=0.045$. The median SII in the positive SARS-CoV-2 PCR group was 780.12 (301.21-2178.90) $\times 10^{3} / \mu \mathrm{L}$. This result was higher than the median in a group with negative SARS-CoV-2 PCR, 584.14 (117.79-1933.87) $\times 10^{3} / \mu \mathrm{L}$. Another research conducted by Patra et al. among the suspected COVID-19 patients admitted to the isolation ward in India showed consistent results. The mean SII in the positive PCR group was $3151.85 \times 10^{3} / \mu \mathrm{L}$, higher than the negative PCR group with a mean of $898.4 \times 10^{3} / \mu \mathrm{L} .{ }^{16}$

Research on the determination of the normal value and reference range of SII has been carried out in China. The overall median was 383.19 (289.18-510.23) $\times 10^{9} /$ L. Median SII in the male group was 377.41 (285.55-498.95) $\times 109 / \mathrm{L}$ and 397.65 (298.43-534.64) $\times 10^{9} / \mathrm{L}$ in the female group. There was a significant difference in the normal value of SII based on gender with $p=0.000 .^{13}$

Systemic immune inflammation index combining three types of blood cells involved in the inflammatory process has shown good results in predicting malignancy with various cut-offs. Increased SII values are usually associated with higher inflammatory responses and decreased immune responses. The meta-analysis conducted by Dong et al. regarding the role of SII in inflammation related to malignancies showed a cut-off of SII that varied between $340-1505 \times 10^{9} / \mathrm{L}^{17}$ Systemic immune inflammation index research on subjects with COVID-19 was also carried out in China by Li et al. using cut-off $1293.11 \times 10^{9} / \mathrm{L}^{18}$

Lagunas-Alvarado et al. showed that the mean SII in subjects with sepsis was $4444.06 \times 10^{9} / \mathrm{L}$, while in subjects without sepsis was $3013.94 \times 10^{9} / \mathrm{L}$. The mean SII was higher in the sepsis group with a value of $p=0.00001 .^{9}$ Luo et al. studied subjects with COVID-19 patients in Wuhan, China, and obtained 
median SII 710 (435-1246) x10\% $/$, subjects who died had significantly higher SII with a median of 1223 (743-1847) $\times 10^{9} / \mathrm{L}$. Subjects who recovered had the median SII of $563(390-882) \times 10^{9} / \mathrm{L}^{19}$ Xue et al. researched 114 patients with COVID-19 and showed elevated SII in a patient with severe disease compared to the mild to moderate patients with SII of $1263.52(752.80-2094.42)$ and 618.35(373.58-1123.39), respectively. ${ }^{20}$

The optimal cut-off values were determined using ROC curves. The area under the curve of SII to predict positive PCR result was 0.630 with $p=0.045$ (95\% CI 0.504 to 0.756) (Fig.1). Thus, the optimal SII values to indicate positive PCR result was $>705 \times 10^{3} / \mu \mathrm{L}$ with sensitivity, specificity, positive predictive value, and negative predictive value of $66.6 \%, 66.6 \%, 56.4 \%$, and $75.5 \%$, respectively.

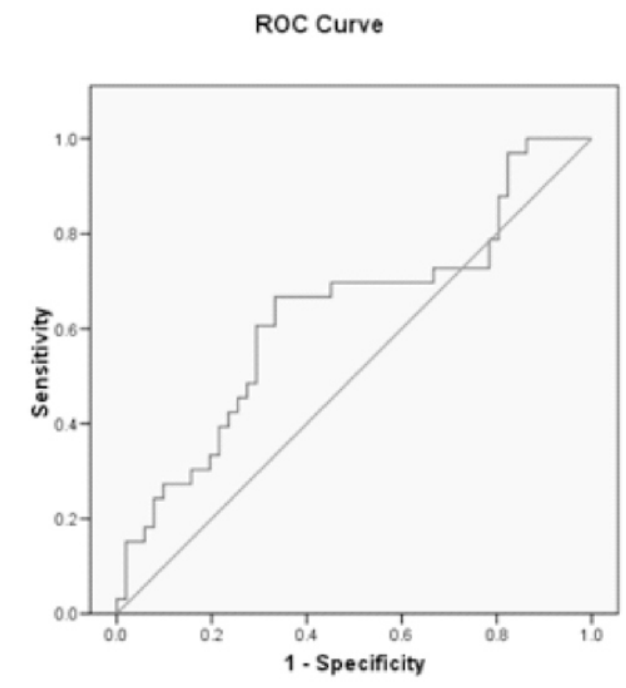

Figure 1. Receiver operating characteristic curve of SII to predict positive PCR result

Table 3. Bivariate analysis of prognostic variables with SARS-CoV-2 PCR result

\begin{tabular}{|c|c|c|c|c|c|c|c|}
\hline \multirow{3}{*}{ Variable } & \multicolumn{4}{|c|}{ Prognosis Classification } & \multirow{3}{*}{ OR } & \multirow{3}{*}{$95 \% \mathrm{CI}$} & \multirow{3}{*}{$\mathbf{p}$} \\
\hline & \multicolumn{2}{|c|}{ PCR Positive } & \multicolumn{2}{|c|}{ PCR Negative } & & & \\
\hline & $\mathrm{n}$ & $\%$ & $\mathrm{n}$ & $\%$ & & & \\
\hline \multicolumn{8}{|l|}{ Gender } \\
\hline Male & 19 & 41.3 & 27 & 58.7 & 1.206 & $0.499-2.916$ & 0.677 \\
\hline Female & 14 & 36.8 & 24 & 63.2 & & & \\
\hline \multicolumn{8}{|c|}{ Hemoglobin (g/dL) } \\
\hline$<12$ & 4 & 40 & 6 & 60 & 1.034 & $0.269-3.984$ & 0.961 \\
\hline$\geq 12$ & 29 & 39.2 & 45 & 60.8 & & & \\
\hline \multicolumn{8}{|c|}{ Erythrocyte $\left(\times 10^{6} / \mu \mathrm{L}\right)$} \\
\hline$<4.5$ & 7 & 53.8 & 6 & 46.2 & 2.019 & $0.613-6.654$ & 0.242 \\
\hline$\geq 4.5$ & 26 & 36.6 & 45 & 63.4 & & & \\
\hline \multicolumn{8}{|c|}{ Leukocyte $\left(\times 10^{3} / \mu \mathrm{L}\right)$} \\
\hline$<5$ & 0 & 0 & 4 & 100 & 1.702 & $1.417-2.045$ & 0.099 \\
\hline$\geq 5$ & 33 & 41.3 & 47 & 58.8 & & & \\
\hline \multicolumn{8}{|l|}{ Platelet $\left(\times 10^{3} / \mu \mathrm{L}\right)$} \\
\hline$<150$ & 1 & 33.3 & 2 & 66.7 & 0.766 & $0.067-8.797$ & 0.830 \\
\hline$\geq 150$ & 32 & 39.5 & 49 & 60.5 & & & \\
\hline \multicolumn{8}{|c|}{ Neutrophil $\left(\times 10^{3} / \mu \mathrm{L}\right)$} \\
\hline$>4.5$ & 23 & 47.9 & 25 & 52.1 & 2.392 & $0.950-6.022$ & 0.061 \\
\hline$\leq 4.5$ & 10 & 27.8 & 26 & 72.2 & & & \\
\hline \multicolumn{8}{|c|}{ Lymphocyte $\left(\times 10^{3} / \mu \mathrm{L}\right)$} \\
\hline$>2.1$ & 13 & 28.9 & 32 & 71.1 & 1.386 & $0.157-0.949$ & $0.036^{*}$ \\
\hline$\leq 2.1$ & 20 & 51.3 & 19 & 48.7 & & & \\
\hline \multicolumn{8}{|c|}{ Eosinophil $\left(\times 10^{3} / \mu \mathrm{L}\right)$} \\
\hline$>0.09$ & 12 & 29.3 & 29 & 70.7 & 0.433 & $0.176-1.066$ & 0.066 \\
\hline$\leq 0.09$ & 21 & 48.8 & 22 & 51.2 & & & \\
\hline \multicolumn{8}{|c|}{ Monocyte $\left(x 10^{3} / \mu L\right)$} \\
\hline$>0.5$ & 22 & 55 & 18 & 45 & 3.667 & $1.455-9.237$ & $0.005^{*}$ \\
\hline$\leq 0.5$ & 11 & 25 & 33 & 75 & & & \\
\hline \multicolumn{8}{|c|}{ Basophil $\left(\times 10^{3} / \mu \mathrm{L}\right)$} \\
\hline$>0.03$ & 11 & 35.5 & 20 & 64.5 & 0.775 & $0.310-1.938$ & 0.585 \\
\hline$\leq 0.03$ & 22 & 41.5 & 31 & 58.5 & & & \\
\hline \multicolumn{8}{|l|}{ SII $\left(\times 10^{3} / \mu \mathrm{L}\right)$} \\
\hline$>705$ & 22 & 56.4 & 17 & 43.6 & 4.000 & $1.580-10.127$ & $0.003^{*}$ \\
\hline$\leq 705$ & & & & & & & \\
\hline
\end{tabular}


Xue et al. also calculated the diagnostic performance of SII in COVID-19 patients. The best cut-off value for SII to predict the severity of the disease was $809.02 \times 10^{9} / \mathrm{L}$ with sensitivity, specificity, negative predictive value, and positive predictive value of $72.41 \%, 67.86 \%, 70.37 \%$, and $70 \%$, respectively. ${ }^{20}$ The results from Xue et al. were consistent with this research. The cut-off value was lower in this research, and the difference in the research population might cause this.

In this study, the risk of positive SARS-CoV-2 PCR was calculated against various hematological parameters, and an SII cut-off of $705 \times 10^{3} / \mu \mathrm{L}$ was used in a subject suspected of being infected with SARS-CoV-2. The results of the bivariate analysis are shown in Table 3.

Significant results were obtained for lymphocyte counts using cut-off $2.1 \times 10^{3} / \mu \mathrm{L}(\mathrm{p}=0.036)$, monocyte counts with cut-off $>0.5 \times 10^{3} / \mu \mathrm{L}(p=0.005)$, and SII using cut off $>705 \times 10^{3} / \mu \mathrm{L}(\mathrm{p}=0.003)$. The odds ratio (OR) of SII $>705 \times 10^{3} / \mu \mathrm{L}$ was 4.00 with $95 \% \mathrm{CI}$ $1.580-10.127$ in suspected COVID-19 subjects to get a positive SARS-CoV-2 PCR result.

A study by Li et al. showed that the cut-off of SII $1293.11 \times 10^{3} / \mu \mathrm{L}$ was able to predict mortality in subjects diagnosed with COVID-19 with a hazard ratio of $2.893,95 \% \mathrm{CI} 1.116-7.222$, and $p=0.028$. The SII hazard ratio to predict the incidence of ARDS in subjects with COVID-19 was equal to $6.832,95 \% \mathrm{CI}$ $2.583-18.074, p<0.001 .^{18}$

Luo et al. compared various biomarkers of infection such as CRP, SII, NLR, procalcitonin, and $\mathrm{D}$-dimer on clinical outcome and disease severity in COVID-19 patients. However, only CRP showed a reasonably good predictive performance for poor clinical outcomes and disease severity. The increase of CRP in subjects who died or were critically ill indicated an excess inflammatory response that was in line with the rise in various proinflammatory cytokines in COVID-19 patients. ${ }^{19}$

The SII calculations reflected the inflammation and immune system activation in the subject with suspected COVID-19. In addition, SII is a relatively inexpensive parameter, easy to perform, and widely available. Therefore, it can be used in the clinical management of patients with suspected SARS-CoV-2 infection.

\section{CONCLUSIONS AND SUGGESTIONS}

A significantly higher median SII was found in subjects with positive SARS-CoV-2 PCR results. In addition, subjects with suspected SARS-CoV-2 infection with elevated SII (> $\left.705 \times 10^{3} / \mu \mathrm{L}\right)$ were at higher risk of positive SARS-CoV-2 PCR results.
The SII can be considered a helpful laboratory parameter for assessing outcomes in subjects with suspected SARS-CoV-2 infection. However, further research with more samples and controlled confounding variables was needed to obtain more valid results.

\section{REFERENCES}

1. Kementerian Kesehatan RI. Pedoman pencegahan dan pengendalian Coronavirus Disesase (COVID-19). Jakarta, Kemenkes, 2020; 17-24.

2. World Health Organization. WHO COVID-19 preparedness and response progress report February to June 30, 2020. Geneva, WHO, 2020; 5-6.

3. Chen G, Wu D, Guo W, Cao Y, Huang D, et al. Clinical and immunological features of severe and moderate coronavirus disease 2019. J Clin Invest, 2020; 130(5): 2620-2629.

4. Henry BM, de Oliveira MHS, Benoit S, Plebani M, Lippi G. Hematologic, biochemical and immune biomarker abnormalities associated with severe illness and mortality in coronavirus disease 2019 (COVID-19): A meta-analysis. Clin Chem Lab Med, 2020; 58(7): 1021-1028

5. Frater JL, Zini G, d'Onofrio G, Rogers HJ. COVID-19 and the clinical hematology laboratory. Int J Lab Hematol, 2020; 42(1): 11-18.

6. Deeks JJ, Dinnes J, Takwoingi Y, Davenport C, Leeflang MMG, et al. Diagnosis of SARS-CoV-2 infection and COVID-19: Accuracy of signs and symptoms; molecular, antigen, and antibody tests; and routine laboratory markers (Protocol). Cochrane Database of Systematic Reviews, 2020; 4: Art. No. CD013596.

7. Chan AS, Rout A. Use of neutrophil-to-lymphocyte and platelet-to- lymphocyte ratios in COVID-19. J Clin Med Res, 2020; 12(7): 448-453.

8. Ustundag Y, Huysal K, Gecgel SK, Unal D. Relationship between $C$-reactive protein, systemic immune-inflammation index, and routine hemogram-related inflammatory markers in low-grade inflammation. Int J Med Biochem, 2018; 1(1): 24-8.

9. Lagunas-Alvarado M, Mijangos-Huesca FJ, Ter'an-Gonz'alez JO, Lagunas-Alvarado MG, Martinez-Zavala M, Reyes Franco I, et al. Systemic immune inflammatory index in sepsis. Med. Int, 2017; 33(3): 303-309.

10. Zhang B, Zhou X, Qiu Y, Feng F, Feng J, et al. Clinical characteristics of 82 death cases with COVID-19. PLoS One, 2020; 15(7): e0235458.

11. Ferrari D, Motta, A, Strollo A, Banfi G, Locatelli M. Routine blood tests as a potential diagnostic tool for COVID-19. Clin Chem Lab Med, 2020; 58(7): 1095-99.

12. Cheng Z, Lu Y, Cao Q, Qin L, Pan Z, et al. Clinical features and chest CT manifestations of Coronavirus Disease 2019 (COVID-19) in a single-center study in Shangai, China. Cardiopulmunary Imaging, 2020; 215: 1-6.

13. Fei $Y$, Wang $X$, Zhang $H$, Huang $M$, Chen $X$, Zhang $C$. 
Reference intervals of systemic immune-inflammation index, neutrophil to lymphocyte ratio, platelet to lymphocyte ratio, mean platelet volume to platelet ratio, mean platelet volume, and red blood cell distribution width-standard deviation in healthy Han adults in Wuhan region in central China. Scandinavian Journal of Clinical and Laboratory Investigation, 2020; 80(6): 500-507.

14. Ruta VM, Man AM, Alexescu TG, Motoc NS, Tamura S, et al. Neutrophil-to-lymphocyte ratio and systemic immune-inflammation index-biomarkers in interstitial lung disease. Medicine, 2020; 56: 381.

15. Wang $Y$, Li Y, Chen $P, X u$ W, Wu Y, Che G. Prognostic value of the pretreatment Systemic Immune-Inflammation Index (SII) in patients with non-small cell lung cancer: A meta-analysis. Ann. Transl. Med, 2019; 7: 10.

16. Patra M, Mahapatra C, Kar PK, Prusty N, Nath PK. Multilineage hematologic pattern involvement in
COVID-19 evaluation from peripheral blood elements. National Journal of Laboratory Medicine, 2021; 10(2): PO09-PO13.

17. Dong M, Shi Y, Yang J, Zhou Q, Lian Y, et al. Prognostic and clinicopathological significance of systemic immune inflammation index in colorectal cancer: A meta-analysis. Ther Adv Med Oncol, 2020; 12: 1-14.

18. Li H, Huang J, Pan W, Zhang C, Chang X, Yang B. Systemic immune-inflammatory index predicts prognosis of patients with COVID-19: A retrospective study. Research Square, 2020; 1-37.

19. Luo X, Zhou W, Yan X, Guo T, Wang B, et al. Prognostic value of $C$-reactive protein in patients with Coronavirus 2019. Clin Infect Dis, 2020; 23: ciaa641.

20. Xue G, Gan X, Wu Z, Xie D, Xiong Y, et al. Novel serological biomarkers for inflammation in predicting disease severity in patients with COVID-19. International Immunopharmacology, 2020; 89: 107065. 